\title{
Tissue Motion Estimation and Correction in Super Resolution Imaging
}

Jensen, Jørgen Arendt; Andersen, Sofie B. ; Hoyos, Carlos A. Villagomez ; Hansen, Kristoffer L.; Sørensen, Charlotte M.; Nielsen, Michael Bachmann

\section{Published in:}

Proceedings of 2019 IEEE International Ultrasonics Symposium

Link to article, DOI:

10.1109/ULTSYM.2019.8925632

Publication date:

2019

Document Version

Peer reviewed version

Link back to DTU Orbit

Citation (APA):

Jensen, J. A., Andersen, S. B., Hoyos, C. A. V., Hansen, K. L., Sørensen, C. M., \& Nielsen, M. B. (2019). Tissue Motion Estimation and Correction in Super Resolution Imaging. In Proceedings of 2019 IEEE International Ultrasonics Symposium (pp. 1107-1110). IEEE. https://doi.org/10.1109/ULTSYM.2019.8925632

\section{General rights}

Copyright and moral rights for the publications made accessible in the public portal are retained by the authors and/or other copyright owners and it is a condition of accessing publications that users recognise and abide by the legal requirements associated with these rights.

- Users may download and print one copy of any publication from the public portal for the purpose of private study or research.

- You may not further distribute the material or use it for any profit-making activity or commercial gain

- You may freely distribute the URL identifying the publication in the public portal 


\title{
Tissue Motion Estimation and Correction in Super Resolution Imaging
}

\author{
Jørgen Arendt Jensen ${ }^{1}$, Sofie B. Andersen ${ }^{2,3}$, Carlos A. Villagomez Hoyos ${ }^{4}$, \\ Kristoffer L. Hansen ${ }^{2}$, Charlotte M. Sørensen ${ }^{3}$, Michael Bachmann Nielsen ${ }^{4}$ \\ ${ }^{1}$ Center for Fast Ultrasound Imaging, Department of Health Technology, \\ Technical University of Denmark, DK-2800 Lyngby, Denmark \\ ${ }^{2}$ Department of Diagnostic Radiology, Rigshospital, Denmark \\ ${ }^{3}$ Department of Biomedical Sciences, University of Copenhagen, \\ ${ }^{4}$ BK Medical, Herlev, Denmark
}

\begin{abstract}
Super resolution imaging (SRI) can attain resolutions in the 10-20 $\mu \mathrm{m}$ range to visualize capillary circulation. It requires completely stationary interrogated tissue during the 3- to 10-minute acquisition, which is not obtainable for live animals apart from in the brain. All other organs move due to respiration, heart pulsation, and muscle activity. SRI can, thus, only be obtained with proper compensation for these motions. A method for full 2D tissue motion estimation is suggested, and it is shown how this motion compensation can restore SRI of small vessels in a rat kidney. A BK 5000 scanner was used with a X18L5 transducer (BK Medical). B-mode and contrast pulse sequence images were acquired with an MI of 0.2 at a $54 \mathrm{~Hz}$ frame rate for $10 \mathrm{~min}$. The left kidney of a male Sprague-Dawley rat was scanned during laparotomy. A 1:10 diluted SonoVue contrast agent (Bracco) was injected through a jugular vein catheter at $100 \mu \mathrm{l} / \mathrm{min}$. Envelope B-mode data were used for $2 \mathrm{D}$ tissue speckle tracking with sub-pixel precision in a central region of the kidney for axial and lateral motion estimation relative to a reference frame. The three motion components from the rat's forced ventilation, heart beat, and residual muscular motion were isolated by using the harmonics of the different motion frequencies (breathing $71 \mathrm{bpm}$, heart $280-350 \mathrm{bpm}$, residual: other frequencies). The precision of the motion was found by aligning signals across cycles and thereby estimating the mean standard deviation. The motion signal was used for compensating the position of the individual bubble locations back to that of the reference frame to remove motion. Estimated peak motions were: Heart: Axial: $1.2 \pm 0.079 \mu \mathrm{m}$, Lateral: $7 \pm 0.99 \mu \mathrm{m}$, Breathing Ax: $8 \pm 0.22 \mu \mathrm{m}$, Lat: $79 \pm 1.64 \mu \mathrm{m}$, and Residual: Ax: $42 \mu \mathrm{m}$, Lat: $110 \mu \mathrm{m}$. The estimation is, thus, sufficiently accurate to correct shifts down to the $10 \mu \mathrm{m}$ capillary level. A motion-corrected image compared to a non-corrected image show small vascular structures in the cortex, which are impossible to separate in the unprocessed image, but can be clearly identified in the motioncorrected image. This demonstrates that motion correction in 2D can enhance SRI quality.
\end{abstract}

\section{INTRODUCTION}

Ultrasound Super Resolution Imaging (SRI) has recently been investigated by a number of research groups [1], [2], [3], [4], [5], [6]. The method uses tracking of micro bubble contrast agents to visualize the micro-vasculature down to vessel sizes of $10-20 \mu \mathrm{m}$, ideally depicting the capillary network. The images are acquired over several minutes, during which the scanned object is considered stationary. This is only possible for fixated objects like a rat brain [5] or a fixated mouse ear [4]. Lately, a number of groups have also addressed motion correction to relax the requirement on stationarity. Hansen et al. [7] showed that motion correction of data from a rat kidney could increase precision from 22 to $8 \mu \mathrm{m}$ for a single vessel. In [8], motion effects were reduced by excluding frames with a too large motion from breathing, yielding a resolution of $2.1 \mu \mathrm{m}$ in the axial direction and $6.1 \mu \mathrm{m}$ in the lateral direction. Two stage motion correction was applied in [9] with a combination of affine registration for the global motion and nonrigid registration for estimating the local tissue deformation. This reduced the width of the micro-vessels by a factor of roughly 1.5 .

The method presented here estimates the full motion for all frames without restrictions on the motion, and then coregister the estimated tracks to a reference frame as described in Section II. The SRI pipeline is investigated on data from the left kidney of a Sprague-Dawley rat. The final results demonstrate that super resolution can be attained, although the motion is 5 to 10 times larger than the precision attained.

\section{MethodS}

\section{A. Data acquisition}

Data were acquired from a healthy male Sprague-Dawley rat according to protocols approved by the Danish National Animal Experiments Inspectorate. Induction of anesthesia was performed with 5 percent isoflurane. After a tracheotomy, the animal was connected to a ventilator with a respiration cycle of 72 respirations/minute. Jugular vein catherization was performed with a polyethylene catheter (PE-10), which was used for injecting the contrast agent, isotonic saline and Nimbex. With the animal in the supine position on a heating pad, the left kidney was exposed through open surgery. The diaphragm was pulled slightly cranially with a retractor to further expose the kidney and reduce respiratory motion. The transducer was placed on the lateral kidney surface and held by a fixated stage. Gel was used for proper interface coupling. The diluted (1:10) contrast agent (SonoVue from Bracco) was injected at $100 \mu \mathrm{l} / \mathrm{min}$, and the 10 -minute data recording 
started when the contrast agent became visible on the scanner display.

A BK 5000 scanner (BK Medical, Herlev, Denmark) acquired the data using a XL18L5 "hockey stick" linear array probe. A B-mode image containing 91 emissions was acquired followed by an contrast pulse sequence with a half pressure emission followed by a full pressure and a half pressure emission for the 91 image lines. The output voltage of the scanner was adjusted to maintain a Mechanical Index (MI) below 0.2. The total number of emissions was 364 and the pulse repetition frequency was $19.6 \mathrm{kHz}$ for a frame rate of $54 \mathrm{~Hz}$. Beamformed RF data from the scanner were stored continuously for the 10 minutes experiment on disc and was later processed in our super resolution pipeline for image generation, motion estimation, motion correction, track generation, and anti-aliased track drawing.

\section{B. Motion estimation}

Motion estimation was performed on the envelope detected B-mode image. A search region was selected in the top part of the kidney image and speckle tracking [10] was performed with a maximum displacement of $\pm 300 \mu \mathrm{m}$ in both the lateral and axial directions. A reference image was selected, and the motion was estimated for the whole data set relative to this image. Using the RF data was also attempted, but the lateral sampling distance was too large to successfully find the lateral motion, and the envelope had to be used.

\section{Motion compensation}

The motion estimates are applied on the bubble locations found by the processing pipeline. Bubble localization was also based on the envelope detected B-mode image by finding local maxima and estimating their centroid location. The time point for the motion depends on their location in the image as a linear sweep of the beams are conducted from the most positive lateral location to the most negative location. The motion estimation gives only one estimates per frame, and the motion time series are, therefore, spline interpolated by a factor of 20 to yield a higher time resolution. A linear interpolation between these time points is then made to match the exact time location corresponding to the spatial location of the bubble. Motion from one frame to the next can easily change with $100-200 \mu \mathrm{m}$, and this time interpolation is therefore essential to attain a co-registration better than $10 \mu \mathrm{m}$.

\section{Image formation and anti-aliasing}

The next step is bubble tracks generation. The bubble position is adjusted by the estimated motion, and the motion corrected bubbles in the next frame are found to determine the bubble closets to the current bubble. The search for the next bubble is restricted to the region given by $v_{s} / f_{r}$, where $v_{s}$ is the maximum allowed bubble velocity and $f_{r}$ is the frame rate of the sequence. The selected bubble is then added to the track and removed from the list of bubbles in the frame. The search continues until no bubble satisfying the criteria is
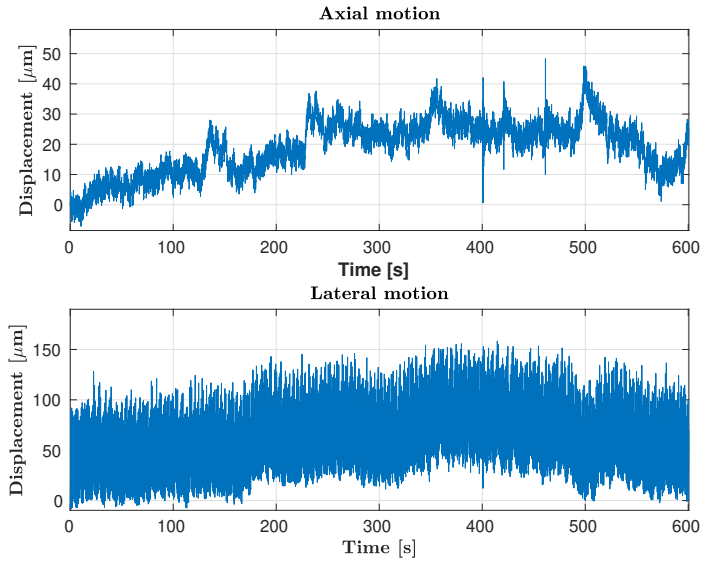

Fig. 1. Motion in the axial (top) and lateral direction (bottom) throughout the full experiment.
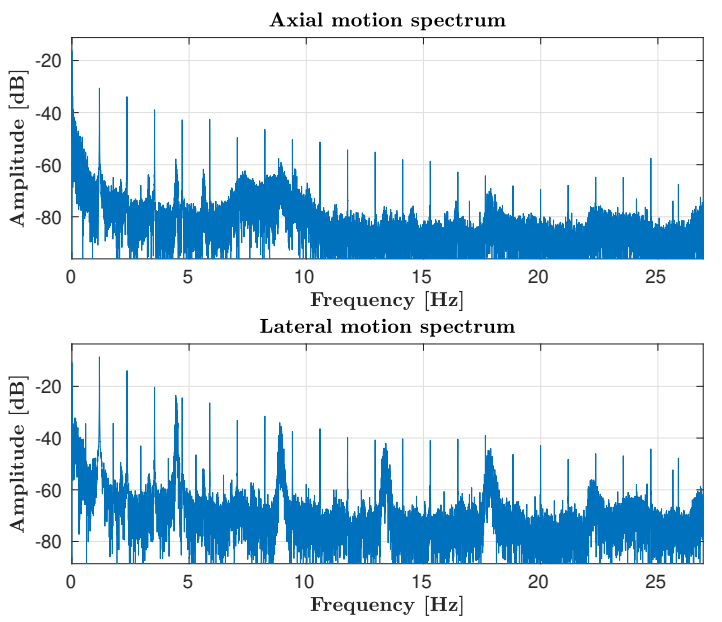

Fig. 2. Spectral decomposition of the motion signal in the axial (top) and lateral direction (bottom).

found. Tracks with less than 3 bubbles are considered outliers from noise and are not stored.

Images are then formed from these tracks by inserting the bubble tracks into a high resolution image. Each track is inserted into the images by drawing an anti-aliased vector between the different positions in the track using the algorithm developed in [11]. The vector is added to the current content of the image, and this finally yields a bubble density image.

\section{RESULTS}

\section{A. Motion estimates and their precision}

The estimated motion from the 10 min experiment is shown in Fig. 1, where the estimated axial motion is shown on the top and the lateral motion on the bottom. The motion consists of three contributing components from the breathing, heart beat, and muscular activity. The components can be separated, as their fundamental frequencies are different, as seen from the spectra of the motion signals shown in Fig. 2. The axial motion spectrum on the top shows narrow and very distinct peaks at frequencies of $m \cdot 70.5 / 60 \mathrm{~Hz}$ where $m$ is the integer harmonic number and $70.5 / 60=1.175 \mathrm{~Hz}$ is the fundamental frequency 

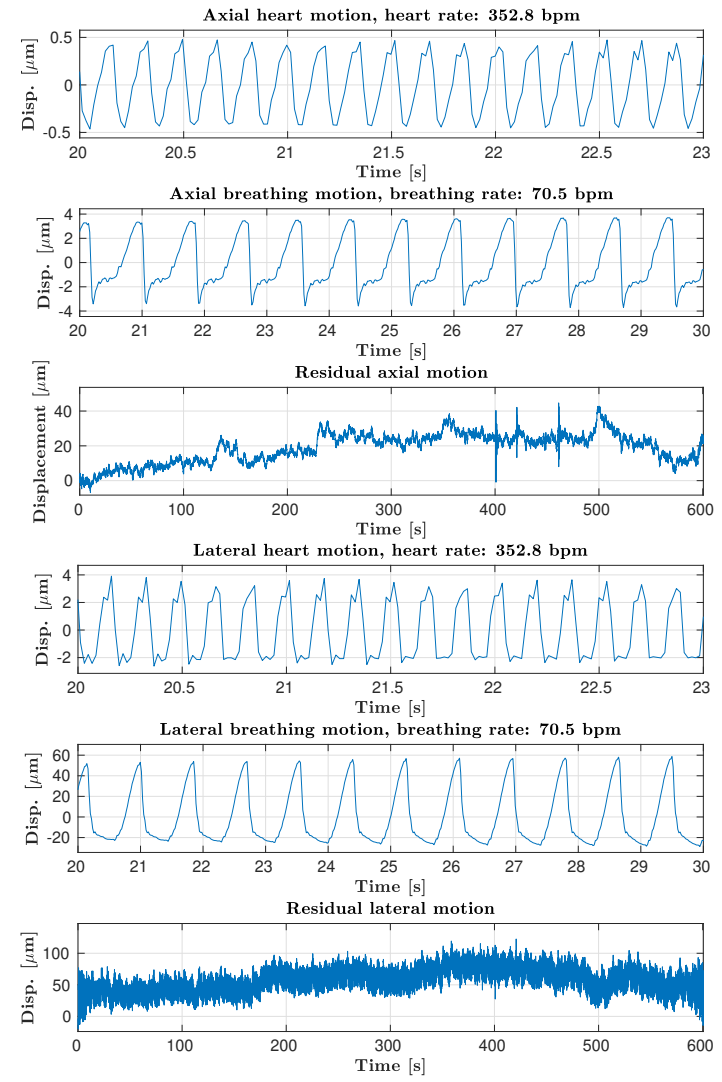

Fig. 3. Separation of the three motion components for the axial motion (top) and the three components for the lateral motion (bottom).

of the forced ventilation. Components up to the 22nd harmonic can be seen in both the axial and lateral spectra (bottom). The peaks are very narrow as the ventilation is mechanically enforced. Components from the heart rate are most easily identified in the lateral motion spectrum at a frequency of $352.8 / 60=5.88 \mathrm{~Hz}$ up to the fifth harmonic. The bandwidth is broader as the heart rate varies over the measurement duration.

Filtration can be used to separate out the various motion signals. Filters only letting the breathing, heart, or muscular motion past have been applied on the spectra, and the six isolated signals are seen in Fig. 3 with axial motion on the top 3 graphs and lateral motions at the bottom. The repetitive patterns for the heart and breathing motion can clearly be seen on the different time scales in the plots. The repetitive motions can be aligned across beats, and this is shown in Fig. 4 for the heart and in Fig. 5 for the breathing. The precision is then estimated by calculating the Standard Deviation (SD) across the waveforms. The largest motion is from the lateral breathing motion with excursions from $-20 \mu \mathrm{m}$ to $+50 \mu \mathrm{m}$ with an SD of $1.6 \mu \mathrm{m}$. The other motions are significantly smaller with SDs below $1 \mu \mathrm{m}$. The combined precision of the motion estimation is therefore likely in the range of a couple of micrometers, which is well below the target of $10 \mu \mathrm{m}$ for detecting and visualizing capillary vessels.
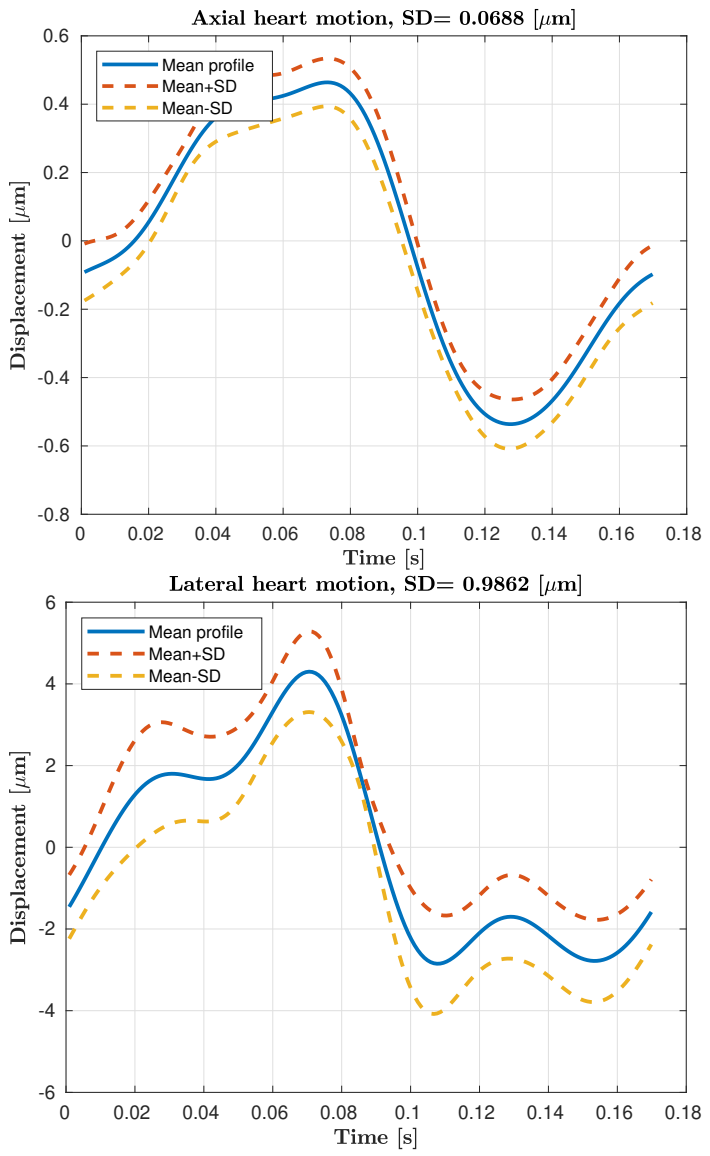

Fig. 4. Mean heart motion for the axial (top) and lateral (bottom) direction.

\section{B. Super resolution images}

The final SR images are shown in Fig. 6. The top image is with a direct insertion of identified bubble positions without making tracks and without anti-aliasing. This has been performed in the lower image, where tracks have been formed from motion compensated data, and anti-aliased vectors from the tracks have been inserted into an imaging matrix with a spatial sampling interval of $10 \mu \mathrm{m}$ in both directions. A clear improvement in image quality can be seen, with the vessels in the cortex more clearly visualized. The vessels in the medulla region are more faint, which is due to the higher intensity of the vessel in the cortex. The dynamic range of the motion compensated image is, thus, larger, and it might be beneficial to use a logarithmic display to capture all details.

\section{DISCUSSION AND CONCLUSION}

The motion of a partly fixated left kidney from a SpragueDawley rat has been estimated using speckle tracking. It was possible to determine the motion with a precision around 1-2 $\mu \mathrm{m}$, which is sufficient to perform motion correction to within the capillary vessel level of $10 \mu \mathrm{m}$. The resolution in the SRI was significantly increased by the use of anti-aliasing when drawing the bubble tracks and performing motion correction. The dense vessel network in the cortical region could be clearly visualized. 

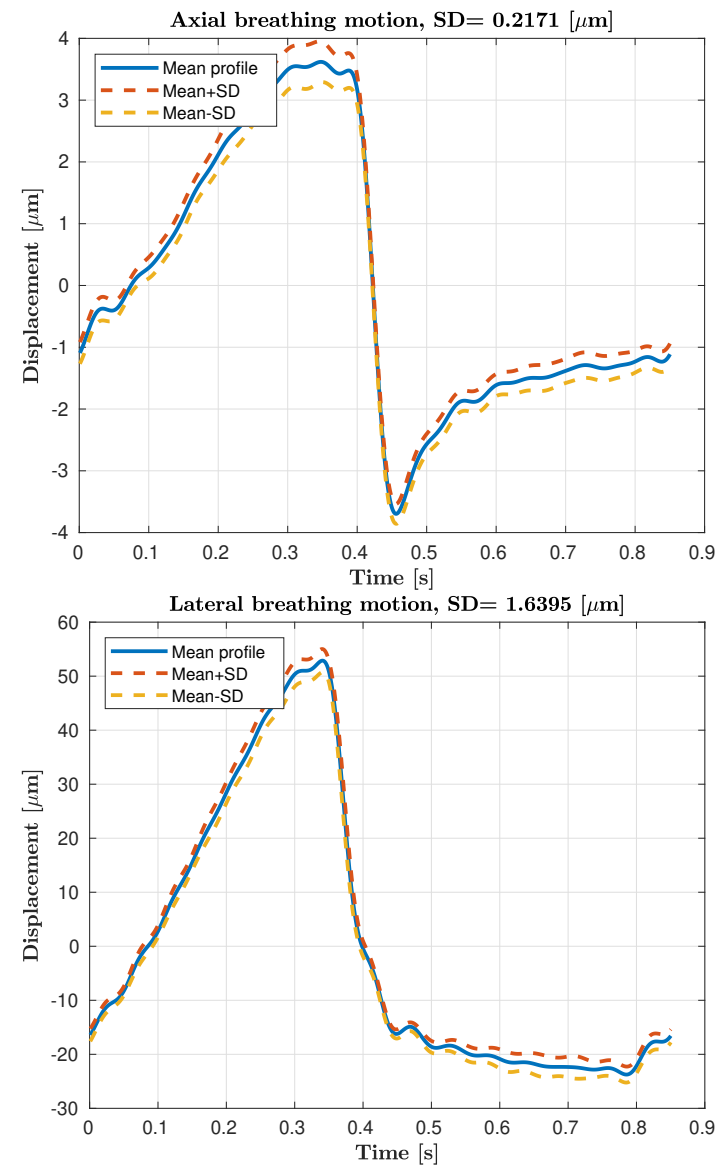

Fig. 5. Mean breathing motion for the axial (top) and lateral (bottom) direction.
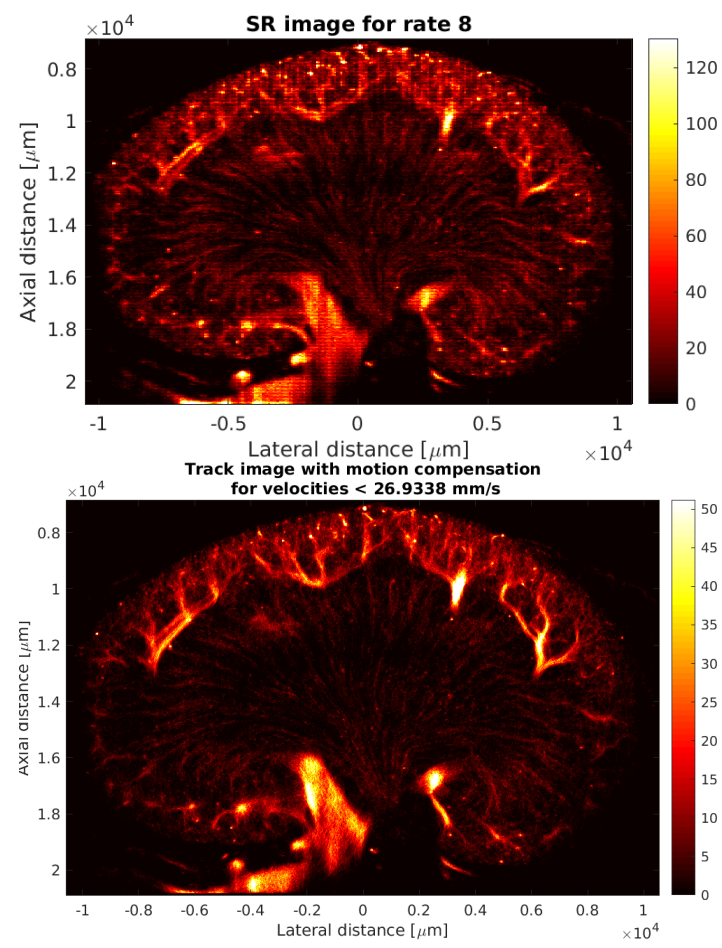

Fig. 6. SR image before interpolation and motion compensation (top) and after motion compensation and anti-aliasing (bottom).
The motion estimation was performed on the envelope data as the lateral sampling density was too small to be useful for lateral motion estimation. The motion precision can, thus, be increased by using more advanced synthetic aperture [12] or plane wave imaging [13].

With the current approach a large zoom on the image show that tracks still have an uncertainty in the 20-30 $\mu \mathrm{m}$ range. The origin of this uncertainty is difficult to determine in-vivo, but is probably due to imprecision in determining the location of the bubbles. This could probably also be improved by using the RF data with a high sampling density or other methods with a better determination of the centroid of the bubbles. The fairly noisy bubble images might also play a significant role here.

\section{ACKNOWLEDGMENT}

This work was financially supported by grant 82-2014-4 from the Danish National Advanced Technology Foundation, by grant 7050-00004B from Innovation Fund Denmark, and from BK Medical, Herlev, Denmark.

\section{REFERENCES}

[1] O. M. Viessmann, R. J. Eckersley, K. C. Jeffries, M. X. Tang, and C. Dunsby, "Acoustic super-resolution with ultrasound and microbubbles," Phys. Med. Biol., vol. 58, pp. 6447-6458, 2013.

[2] M. A. O'Reilly and K. Hynynen, "A super-resolution ultrasound method for brain vascular mapping," Med. Phys., vol. 40, no. 11, pp. 110701-7, 2013.

[3] Y. Desailly, J. Pierre, O. Couture, and M. Tanter, "Resolution limits of ultrafast ultrasound localization microscopy," Phys. Med. Biol., vol. 60, no. 22 , pp. 8723-8740, 2015.

[4] K. Christensen-Jeffries, R. J. Browning, M. Tang, C. Dunsby, and R. J. Eckersley, "In vivo acoustic super-resolution and super-resolved velocity mapping using microbubbles," IEEE Trans. Med. Imag., vol. 34, no. 2, pp. 433-440, February 2015.

[5] C. Errico, J. Pierre, S. Pezet, Y. Desailly, Z. Lenkei, O. Couture, and M. Tanter, "Ultrafast ultrasound localization microscopy for deep superresolution vascular imaging," Nature, vol. 527, pp. 499-502, November 2015.

[6] D. Ackermann and G. Schmitz, "Detection and tracking of multiple microbubbles in ultrasound B-mode images," IEEE Trans. Ultrason., Ferroelec., Freq. Contr., vol. 63, no. 1, pp. 72-82, January 2016.

[7] K. B. Hansen, C. A. Villagomez-Hoyos, J. Brasen, K. Diamantis, V. Sboros, C. M. Sørensen, and J. A. Jensen, "Robust microbubble tracking for super resolution imaging in ultrasound," in Proc. IEEE Ultrason. Symp., 2016, pp. 1-4.

[8] J. Foiret, H. Zhang, T. Ilovitsh, L. Mahakian, S. Tam, and K. W. Ferrara, "Ultrasound localization microscopy to image and assess microvasculature in a rat kidney," Scientific Reports, vol. 7, no. 1, pp. 13662:1-12, 2017.

[9] S. Harput, K. Christensen-Jeffries, J. Brown, Y. Li, K. J. Williams, A. H. Davies, R. J. Eckersley, C. Dunsby, and M. Tang, "Two-stage motion correction for super-resolution ultrasound imaging in human lower limb," IEEE Trans. Ultrason., Ferroelec., Freq. Contr., vol. 65 , no. 5, pp. 803-814, 2018.

[10] G. E. Trahey, J. W. Allison, and O. T. von Ramm, "Angle independent ultrasonic detection of blood flow," IEEE Trans. Biomed. Eng., vol. BME-34, no. 12, pp. 965-967, 1987

[11] X. Wu, "An efficient antialiasing technique," Computer Graphics, vol. 25 , no. 4, pp. 143-152, 1991.

[12] J. Jensen, J. B. Olesen, M. B. Stuart, P. M. Hansen, M. B. Nielsen, and J. A. Jensen, "Vector velocity volume flow estimation: Sources of error and corrections applied for arteriovenous fistulas," Ultrasonics, vol. 70, pp. 136-146, 2016.

[13] M. Tanter and M. Fink, "Ultrafast imaging in biomedical ultrasound," IEEE Trans. Ultrason., Ferroelec., Freq. Contr., vol. 61, no. 1, pp. 102119, January 2014. 